\title{
DESCRIÇÃO DAS ALTERAÇÕES CROMOSSÔMICAS EM PORTADORES DE MIELOMA MÚLTIPLO ACOMPANHADOS NO CENTRO DE TRANSPLANTE DO HOSPITAL PORTUGUÊS DE SALVADOR, BAHIA.
}

\author{
Description of chromosomal abnormalities in patients bearers of multiple myeloma followed up \\ at the Bone Marrow Transplant Center of the Hospital Portugues in Salvador, Bahia.
}

Ronald Sérgio Pallotta Filho',2, Mônica Conchon Ribeiro de Mello ${ }^{3}$

\begin{abstract}
RESUMO
Nos últimos anos tem ocorrido uma revolução no conhecimento referente à patogênese do mieloma múltiplo. A incorporação da citogenética e da biologia molecular têm possibilitado a determinação de alterações cromossômicas por vezes relacionadas a diferentes subgrupos da doença, contribuindo como marcadores biológicos e de prognóstico para a tomada de decisões clínicas. Objetivo: Descrever as alterações cromossômicas em pacientes portadores de mieloma múltiplo acompanhados no Centro de Transplante de Medula Óssea do Hospital Português em Salvador, Bahia. Métodos: Estudo descrito de corte transversal a partir da revisão de prontuários de pacientes portadores de mieloma múltiplo acompanhados de 2001 a 2006 no Centro que rotineiramente ao diagnóstico e/ou durante o tratamento tiveram a doença avaliada a partir da coleta de medula óssea para análise citogenética, histológica e imuno-histoquímica para estimar o comprometimento do local. Resultados: Dos 39 pacientes viáveis para análise, em 51\% dos casos não foram obtidas metáfases, em 36\% observou-se cariótipo sem anormalidade e em 13\% foram encontradas alterações. A correlação entre o estudo citogenético e a atividade da patologia nos pacientes demonstrou razão de prevalência igual a 1,8 entre grupos, enquanto a correlação entre alterações citogenéticas no grupo de pacientes com doença em atividade a razão de prevalência foi de 5,5. Conclusão: Desta forma, a despeito do baixo índice mitótico da doença, os autores confirmam ser a citogenética uma ferramenta adequada para determinar o prognóstico principalmente quando realizada em pacientes com doença em atividade, devendo esta fazer parte do estudo inicial dos pacientes com mieloma múltiplo, podendo auxiliar na estratégia terapêutica a ser definida.
\end{abstract}

Descritores: Mieloma Múltiplo; Citogenética, Prognóstico.

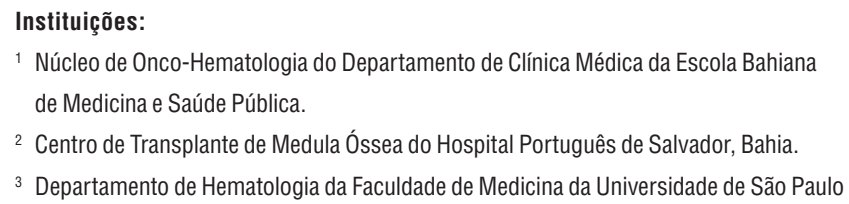

\section{INTRODUÇÃO}

Mieloma múltiplo (MM) é uma doença maligna dos plasmócitos que acomete pacientes próximos da sexta década de vida. É responsável por 1\% de todos os tipos de câncer e por 10\% das neoplasias hematológicas, sendo mais freqüente em negros do que em brancos e os homens são mais afetados do que mulheres. ${ }^{1}$ Para melhor compreensão de seu comportamento biológico, se faz necessário o estudo das características intrínsecas do plasmócito maligno e sua relação com o microambiente medular.

Nos últimos anos tem ocorrido uma revolução no conhecimento referente à patogênese do MM. A incorporação da citogenética e da biologia molecular têm possibilitado a determinação de alterações cromossômicas por vezes relacionadas a diferentes subgrupos da doença, contribuindo como marcadores biológicos e de prognóstico para a tomada de decisões clínicas. ${ }^{2-4}$

Baseado na carência de informações na literatura médica brasileira referente a esse assunto, o presente estudo tem como objetivo descrever a prevalência das alterações cromossômicas 
em pacientes portadores de mieloma múltiplo acompanhados no Centro de Transplante de Medula Óssea do Hospital Português em Salvador.

\section{CASUÍSTICA E MÉTODO}

Desenho: Estudo observacional descritivo de corte transversal.

População estudada: Pacientes com mieloma múltiplo (MM) acompanhados de 2001 a 2006 no Centro de Transplante de Medula Óssea do Hospital Português, em parceria com o Núcleo de Onco-Hematologia da Escola Bahiana de Medicina e Saúde Pública-Fundação Bahiana para o Desenvolvimento das Ciências (EBMSP-FBDC).

Critérios de inclusão: A partir da revisão de prontuários, foram inclusos pacientes que apresentavam informações clínicas e laboratoriais suficientes para confirmação diagnóstica de MM, bem como estudo anatomo-patológico, imuno-histoquímico e citogenético realizado em algum momento do tratamento.

Protocolo de estudo: Pacientes portadores de mieloma múltiplo no Centro de Transplante de Medula Óssea do Hospital Português rotineiramente ao diagnóstico e/ou durante o tratamento tiveram a doença avaliada a partir da coleta da medula óssea para análise citogenética, histológica e imuno-histoquímica para estimar o comprometimento do local.

Para os testes anatomo-patológicos e imuno-histoquímicos foram retirados da medula óssea fragmentos a partir da espinha ilíaca póstero-superior dos pacientes por agulha de Jamishid sob anestesia local, de acordo com técnicas convencionais. Em seguida, as amostras foram enviadas para avaliação a um único patologista. $\mathrm{O}$ estudo histológico convencional foi complementado por painel imuno-histoquímico previamente determinado com marcadores para CD 34 (células precursoras, marca Dako/cloneQBEND-10), CD20 (linfócitos B, marca Dako/clone-L26), CD3 (linfócitos T, marca Dako/clone-LEU4), CD68 (macrófagos, neutrófilos e monócitos, marca Dako/clone-PMG1) e VS38 (plasmócitos, marca Dako/clone-VS38), além da análise de clonalidade através das frações Kappa (marca Dako/clone R10-21) e Lambda (marca Dako/clone N10-2).

O material para estudo de citogenética clássica foi coletado no mesmo momento da biopsia e analisado no laboratório especializado em genética da Faculdade de Medicina da Universidade de São Paulo. As análises foram realizadas a partir de culturas com RPMI 1640, soro fetal bovino, L-glutamina e antibiótico (penicilina/ estreptomicina) e como agente estimulante o phorbol 13-acetate (TPA) por três a cinco dias.

Esses exames, que tiveram por finalidade avaliar alterações cromossômicas e do comprometimento da medula óssea por infiltração de plasmócitos tumorais, puderam informar a atividade ou não da doença no microambiente medular. Tais dados estavam anexados aos prontuários dos pacientes que foram revisados no presente estudo.

Com relação à atividade da doença, dividimos a população em dois grupos:

- Grupo que não apresentava qualquer sinal de atividade, atingindo remissão completa com ausência de proteína M no soro e urina por imunofixação e sem infiltração de plasmócitos clonais na medula óssea.
- Grupo com qualquer sinal de atividade da doença, seja na analise imunohistologica ou laboratorial.

\section{RESULTADOS}

Dos 68 pacientes acompanhados, foram analisados 39 pacientes que cumpriam os critérios de inclusão principalmente em relação ao estudo citogenético onde 10 realizaram pré-tratamento e 29 durante o tratamento. Desse grupo, 59\% eram do sexo feminino, $64 \%$ não brancos e a mediana de idade foi 53 anos. Em relação ao estadiamento em que se encontrava a doença ao diagnóstico, 95\% eram EC III A e 5\% III B de acordo com a classificação de Durie e Salmon, potencialmente devido a grande dificuldade de acesso a atendimento especializado na região.

Analisando os tipos diferentes de imunoglobulinas secretadas ao diagnóstico, verificou-se que $67 \%$ dos pacientes eram secretores de $\mathrm{IgG}, 18 \%$ de $\operatorname{IgA}, 5 \%$ dos pacientes as células do mieloma produziam somente cadeias leves e $10 \%$ não tiveram cadeias identificadas por imunoeletroforese de proteínas no soro e urina pelo método de imunofixação como apresentado resumidamente na Tabela 1.

Tabela 1. Características epidemiológicas dos pacientes portadores de Mieloma Múltiplo , acompanhados de 2001 a 2006 no Centro de Transplante de Medula Óssea do Hospital Português

\begin{tabular}{|c|c|}
\hline CARACTERÍSTICAS & VALORES \\
\hline $\begin{array}{ll}\text { Mediana } & \text { Idade }\end{array}$ & 53 \\
\hline $\begin{array}{l}\text { Variação } \\
\text { Feminino }\end{array}$ & $\begin{array}{l}34-66 \\
N^{0} .(\%) \\
23(59)\end{array}$ \\
\hline Masculino & $16(41)$ \\
\hline Brancos & $14(36)$ \\
\hline Não Brancos & $25(64)$ \\
\hline $\lg G$ & $26(67)$ \\
\hline $\lg A$ & $7(18)$ \\
\hline Cadeia leve & $2(5)$ \\
\hline Estáqio ao diagnóstico & \\
\hline $\begin{array}{l}\text { IIIA } \\
\text { IIIB }\end{array}$ & $\begin{array}{c}37(95) \\
2(5)\end{array}$ \\
\hline
\end{tabular}

IgG=imunoglobulina $G, \lg A=$ imunoglobulina $A$, III A=estádio clinico de DurieSalmon com grande massa tumoral caracterizada por envolvimento ósseo, hemoglobina $<10 \mathrm{~g} / \mathrm{dl}$, cálcio > 12 e cretinina < que $2 \mathrm{mg} / \mathrm{dl}$, IIIB = estádio clinico de Durie-Salmon com grande massa tumoral caracterizada por envolvimento ósseo, hemoglobina <10 $\mathrm{g} / \mathrm{dl}$, cálcio > 12 e cretinina > que $2 \mathrm{mg} / \mathrm{dl}$

Em $51 \%$ dos 39 casos analisados não foram obtidas metáfases, em $36 \%$ observou-se cariótipo sem anormalidade e em 13\% foram encontradas alterações.

A deleção envolvendo o cromossomo13, del (13)(q14.3) foi mais freqüente, tendo sido encontrada em 4 dos 39 pacientes (10\%). 
Desses, dois (5\%) apresentavam apenas alteração envolvendo o cromossomo 13 e dois (5\%) possuíam deleção do cromossomo 13, del (13)(q14.3) acrescida de translocação envolvendo o cromossomo 14, t(14q32). A deleção do cromossomo 6, del (6) (q15q22) foi detectada em um (3\%) dos pacientes (gráfico 1).

Gráfico 1. Análise dos 39 pacientes portadores de Mieloma Multiplo acompanhados de 2001 a 2006 pelo Centro de Transplante de Medula Óssea do Hospital Português que realizaram estudo citogenético.

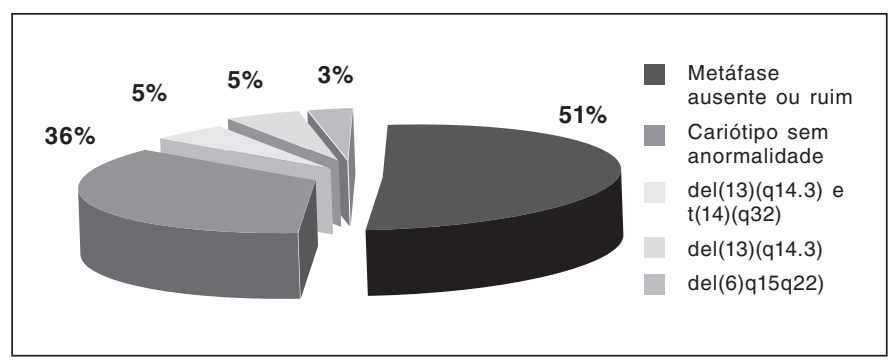

del $=$ deleção, $t=$ translocação

Todos os pacientes que foram analisados quanto ao cariótipo também realizaram avaliação histológica e imuno-histoquímica da medula óssea para estimar a parcela de envolvimento por plasmócitos neoplásicos. Desta forma, foi verificada ausência de infiltração em 28 (72\%) dos pacientes no momento que o cariótipo foi coletado, e infiltração em 11 (28\%) pacientes.

A correlação entre o estudo citogenético e a atividade da doença nos pacientes demonstrou que dos 11 pacientes que não apresentavam remissão completa, oito obtiveram metáfases avaliáveis. Por outro lado, dos 28 pacientes que apresentavam remissão completa (pacientes com ausência de proteína M no soro e urina por imunofixação e sem infiltração de plasmócitos clonais na MO), 11 tiveram metáfases avaliáveis. (Tabela 2).

Tabela 2. Determinação da presença de metáfase em relação à atividade da doença

\begin{tabular}{|l|c|c|}
\hline & Com metáfase & Sem metáfase \\
\hline Doença sem remissão completa & 8 & 3 \\
\hline Doença em remissão completa & 11 & 17 \\
\hline
\end{tabular}

Desta forma, os dados demonstraram que a prevalência de metáfase no grupo de pacientes com doença em atividade (sem remissão completa) foi de $72 \%$, enquanto que no grupo com doença em remissão completa foi de 39,3\%. Sendo assim, podemos concluir que a razão de prevalência foi de 1,8 entre os grupos.

A análise do grupo de 19 pacientes com metáfases avaliáveis demonstrou que dos 5 pacientes com alterações estruturais ou numéricas, quatro apresentavam doença em atividade, ou seja, sem remissão completa. Quando analisados os 14 pacientes que não apresentavam alterações citogenéticas, quatro demonstravam sinais de atividade de doença (Tabela 3 ).
Tabela 3. Determinação da presença de alterações citogenéticas em relação à atividade da doença na população estudada

\begin{tabular}{|l|c|c|}
\hline & $\begin{array}{c}\text { Com alterações } \\
\text { citogenéticas }\end{array}$ & $\begin{array}{c}\text { Sem alterações } \\
\text { citogenéticas }\end{array}$ \\
\hline Doença sem remissão completa & 4 & 4 \\
\hline Doença em remissão completa & 1 & 10 \\
\hline
\end{tabular}

Desta forma, os dados demonstraram que a prevalência de alterações citogenéticas no grupo de pacientes com doença em atividade, ou seja, sem remissão completa, foi de $50 \%$, enquanto que no grupo com doença em remissão completa foi de $9 \%$. Sendo assim podemos concluir que a razão de prevalência foi de 5,5 entre os grupos.

\section{DISCUSSÃO}

O estudo do MM por citogenética é muito complexo, sendo dificultado principalmente pelo baixo índice mitótico da doença, conseguindo-se metáfases em cerca $20 \%$ a $30 \%$ dos casos. Porém, é possível subdividir as alterações encontradas em 2 categorias principais, uma chamada hiperdiploide, definida pela presença de inúmeras trissomias e baixa freqüência de translocações IgH que contrasta com a categoria não-hiperdiploide, esta definida pela alta freqüência de translocações $\operatorname{IgH}$ e freqüente perda cromossômica, em especial dos cromossomos 13,14, 17 e 8.,6

Em nosso estudo, conseguimos obter metáfases em $49 \%$ dos casos. Porém, vale destacar que desses 19 casos, 14 demonstraram cariótipo sem anormalidade, o que deve ser discutido, uma vez que a técnica não permite diferenciar as células tumorais das restantes populações celulares. Isto leva muitas vezes a resultados falsos negativos baseados na análise cariotípica de células mielóides em divisão, presentes na medula óssea. Outro dado importante é quanto ao momento em que o cariótipo foi coletado, já que nos pacientes em que a citogenética foi coletada com doença em atividade, além de maior chance de obter metáfases $(8 / 11)$, também se observou maior freqüência de alterações $(50 \%)$. Tais dados reforçam a importância de se coletar estudo citogenético para o diagnóstico.

Quando analisamos os 13\% em que foram encontradas alterações, observamos deleção envolvendo o cromossomo 13, del (13)(q14.3) como a mais freqüente, sendo encontrada em quatro dos 39 pacientes (10\%). Tal alteração apresenta uma alta prevalência no MM e em outras doenças de células plasmocitárias. ${ }^{7,8}$ Utilizando-se técnicas citogenéticas, a -13q14 pode ser detectada em $10 \%$ a $20 \%$ dos pacientes com MM, sendo que, com a incorporação da técnica de FISH, essa porcentagem pode aumentar para $40 \%$ a $50 \% .{ }^{11-13}$

A -13q14 é um fator prognóstico desfavorável independente associado à menor sobrevida e resposta terapêutica, independentemente do tipo de tratamento (quimioterapia convencional ou de altas doses) ou método de detecção utilizado (citogenética convencional ou FISH). ${ }^{12-17}$ Contudo, é importante ressaltar que o efeito da -13q14 na sobrevida é mais desfavorável quando detectada por citogenética do que quando detectada por FISH. ${ }^{12-17}$ Esse achado deve-se aos efeitos aditivos no prognóstico de detecção de metáfases anormais, o que indica maior carga tumoral e um clone em fase proliferativa, relacionados com estágio mais avançado da doença. 
Achados adicionais envolvendo o cromossomo 14 também são descritos na literatura, em especial aqueles associados ao lócus gênico $14 q 32$, que é a mais freqüente anormalidade estrutural comprometendo cerca de $60 \%$ de todas as translocações do $\operatorname{IgH}$. A presença de parceiros heterogêneos tem sido descrita, como por exemplo, na forma de $\mathrm{t}(11 ; 14)(\mathrm{q} 13 ; \mathrm{q} 32)$ que apresenta conseqüências biológicas favoráveis associadas à hiperexpressão da ciclina D1, que contrasta com $\mathrm{t}(4 ; 14)$ e a $\mathrm{t}(14 ; 16)$ associadas a um prognóstico altamente desfavorável. ${ }^{5}$

Com relação à alteração do cromossomo 6 encontrada, pode-se discutir apenas como esta enriquece a grande diversidade de locus cromossômicos envolvidos nas translocações das imunoglobulinas. Sua relação com o prognóstico é discutível, estando geralmente envolvida na translocação 14q32 de pacientes com MM, que pode ter evolução favorável. ${ }^{5}$

Desta forma, baseado na alteração citogenética como valor prognóstico diferencial, neste estudo encontram-se principalmente pacientes com marcadores citogenéticos definidos como de alto risco pela mSMART ${ }^{18}$ e que em geral acometem cerca de $25 \%$ dos pacientes, entre elas a não-hiperdiploidia, a deleção citogenética envolvendo os cromossomos 13 e 14. Embora não encontradas neste estudo, a t $(4 ; 14), \mathrm{t}(14 ; 16)$ e/ou a del 17 p são o subgrupo de alterações de pior prognóstico. Não foram encontradas outras alterações definidas como risco "standard" de prognóstico pela mSMART, em geral mais freqüentes acometendo cerca de $75 \%$ dos pacientes, onde as alterações hiperdiploides e as t $(11 ; 14)$ e t $(6 ; 14)$ associadas à beta 2 microglobulina com níveis inferiores a 5,5 e níveis de desidrogenase lática normal estão inclusas.

\section{CONCLUSÃO}

Neste estudo, os resultados foram condizentes com os achados da literatura em relação à obtenção de metáfases, porém contrastam quanto à prevalência de alteração citogenética (13\%) e quanto ao predomínio dessas alterações, onde na maioria foram consideradas de alto risco, de acordo com a mSMART classification. Porém, considerando-se que nem todos os pacientes tiveram o cariótipo coletado ao diagnóstico, deve-se avaliar esses dados com cautela. A despeito do baixo índice mitótico da doença, os autores confirmam ser a citogenética uma ferramenta adequada para determinar o prognóstico, principalmente quando realizada em pacientes com doença em atividade, devendo esta fazer parte do estudo inicial dos pacientes com MM podendo auxiliar na estratégia terapêutica a ser definida.

\section{ABSTRACT}

In recent years, there has been a revolution in the knowledge concerning the pathogenesis of multiple myeloma. The incorporation of cytogenetics and molecular biology has allowed the determination of chromosomal changes sometimes related to different subgroups of the disease, contributing as biological markers of prognosis and for making clinical decisions Purpose: To describe the chromosomal abnormalities in patients with multiple myeloma at the Bone Marrow Transplant Center of the Hospital Portugues in Salvador, Bahia. Methods: A cross-section descriptive study from medical records of patients with multiple myeloma followed from 2001 to 2006 at the Center, who routinely as part of the diagnostic procedures and/or along the treatment had the disease assessed by the bone marrow collection for cytogenetic, histological and immunohistochemical analysis to assess the involvement of the site. Results: From 39 patients able to be analyzed, in $51 \%$ of cases there were no metaphases, in $36 \%$ were observed no abnormal karyotype, and in $13 \%$ was found changes. Correlation between viability of the cytogenetic analysis and rate of active disease in patients showed a prevalence as much as 1.8 , while the correlation between cytogenetic changes in patients with active disease, the prevalence rate was 5.5. Conclusion: Therefore, despite the low mitotic index of the disease, the authors confirmed that cytogenetic is an appropriate tool to set the prognosis, especially when performed in patients with active disease, and this should be part of the initial study of patients with multiple myleloma, and it may help the therapeutic strategy to be defined.

Keywords: Multiple Myeloma; Cytogenetics, Prognosis

\section{REFERÊNCIAS}

1. Sirohi B, Powles R. Multiple Myeloma. Lancet. 2004;363:875-87.

2. Dewald G, Kyle R, Hicks G, et al. The clinical significance of cytogenetic studies in 100 patients with multiple myeloma, plasma cell leukemia, or amyloidosis. Blood. 1985;66:380-90.

3. Tabernero D, Miguel JS, Garcia-Sanz M, et al. Incidence of chromosome numerical changes in multiple myeloma: fluorescence in situ hybridization analysis using 15 chromosome-specific probes. Am J Pathol. 1996;149:153-61.

4. Zandecki M, Lai J, Facon T. Multiple myeloma: almost all patients are cytogenetically abnormal. Br J Haematol. 1996;94:217-27.

5. Fonseca R, Barlogie B, Bataille B, Bastard C, Bergsagel PL, Chesi M, et al Genetics and cytogenetics of multiple myeloma : a workshop report. Cancer Res. 2004;64:1546-58.
6. Fonseca R, Debes-Marun CS, Picken EB, Dewalt JW, Bryant SC, Winkler JM, et al. The recurrent IgH translocation are highly associated with non-hyperdiploid variant of multiple myeloma. Blood. 2003;102:2562-7.

7. Avet-Loiseau H, Li J, Morineau N, et al. Monosomy 13 is associated with the transition of monoclonal gammopathy of undetermined significance to multiple myeloma. Blood. 1999;94:2.583-9.

8. Drach J, Kaufmann H, Urbauer E, et al. The biology of multiple myeloma. J. Cancer Res. Clin. Oncol. 2000;126:441-7.

9. Sawyer J, Waldron J, Jagannath S, et al. Cytogenetic findings in 200 patients with multiple myeloma. Cancer Genet Cytogenet. 1995;82:41-9.

10. Dao D, Sawyer J, Epstein J, et al. Deletion of the Retinoblastoma gene in multiple myeloma. Leukemia 1994;8:1.280-4. 
11. Avet-Loiseau H, Facon T, Daviet A, et al. 14q32 translocations and monosomy 13 observed in monoclonal gammopathy of undetermined significance delineate a multistep process for the oncogenesis of multiple myeloma. Cancer Research. 1999;59:4.546-50

12. Zojer N, Konigsberg R, Ackermann J, et al. Deletion of 13 q14 remains an independent adverse prognostic variable in multiple myeloma despite its frequent detection by interphase fluorescence in situ hybridization. Blood. 2000;95:1.925-30.

13. Fonseca R, Harrington D, Oken M, et al. Biologic and prognostic significance of interphase FISH detection of chromosome 13 abnormalities in multiple myeloma: an Eastern Cooperative Oncology Group (ECOG) Study. Cancer Research 2002;62:715-20.

14. Facon T, Avet-Loiseau H, Guillerm G, et al. Chromosome 13 abnormalities identified by FISH analysis and serum beta-2-microglobulin produce a powerful myeloma staging system for patients receiving high-dose therapy. Blood. 2001;97:1.566-71.

15. Desikan R, Barlogie B, Sawyer J, et al. Results of high-dose therapy for 1000 patients with multiple myeloma: durable complete remissions and superior survival in the absence of chromosome 13 abnormalities. Blood. 2000;95:4.008-10.

16. Shaughnessy J, Tian E, Sawyer J, et al. Prognostic impact of cytogenetic and interphase fluorescence in situ hybridization-defined chromosome 13 deletion in multiple myeloma: early results of total therapy II. Br J Haematol. 2003;120:44-52.

17. Seong C, Delasalle K, Hayes K, et al. Prognostic value of cytogenetics in multiple myeloma. Br J Haematol. 1998;101:189-94.

18. Dispenzierii A, Rajukmar SV, Gertz MA, Fonseca R, Lacy MO, Bergsagel PL, et al. Treatment of newly diagnosed multiple myeloma based on Mayo Classification of Myeloma and Risk-Adapted Therapy (mSMART). Mayo Clin Proc. 2007;82:323-41 